\title{
Asteroid Discovery and Characterization with the Large Synoptic Survey Telescope
}

\author{
R. Lynne Jones ${ }^{1}$, Mario Jurić ${ }^{2}$ and Željko Ivezić ${ }^{3}$ \\ ${ }^{1}$ University of Washington, email: 1 jones@astro.washington.edu \\ ${ }^{2}$ University of Washington, email: mjuric@astro.astro.washington.edu \\ ${ }^{3}$ University of Washington, email: ivezic@astro.astro.washington.edu
}

\begin{abstract}
The Large Synoptic Survey Telescope (LSST) will be a ground-based, optical, allsky, rapid cadence survey project with tremendous potential for discovering and characterizing asteroids.

With LSST's large $6.5 \mathrm{~m}$ diameter primary mirror, a wide 9.6 square degree field of view 3.2 Gigapixel camera, and rapid observational cadence, LSST will discover more than 5 million asteroids over its ten year survey lifetime. With a single visit limiting magnitude of 24.5 in $r$ band, LSST will be able to detect asteroids in the Main Belt down to sub-kilometer sizes. The current strawman for the LSST survey strategy is to obtain two visits (each 'visit' being a pair of back-to-back 15 s exposures) per field, separated by about 30 minutes, covering the entire visible sky every 3-4 days throughout the observing season, for ten years.

The catalogs generated by LSST will increase the known number of small bodies in the Solar System by a factor of 10-100 times, among all populations. The median number of observations for Main Belt asteroids will be on the order of 200-300, with Near Earth Objects receiving a median of 90 observations. These observations will be spread among ugrizy bandpasses, providing photometric colors and allow sparse lightcurve inversion to determine rotation periods, spin axes, and shape information.

These catalogs will be created using automated detection software, the LSST Moving Object Processing System (MOPS), that will take advantage of the carefully characterized LSST optical system, cosmetically clean camera, and recent improvements in difference imaging. Tests with the prototype MOPS software indicate that linking detections (and thus 'discovery') will be possible at LSST depths with our working model for the survey strategy, but evaluation of MOPS and improvements in the survey strategy will continue. All data products and software created by LSST will be publicly available.
\end{abstract}

Keywords. surveys, catalogs, minor planets, asteroids

\section{Introduction}

The Large Synoptic Survey Telescope (LSST) is a next-generation survey project, coupling a world-class telescope facility with cutting-edge data management software and calibration efforts. Its primary science drivers are to constrain dark matter and dark energy, to map the Milky Way and Local Volume, to catalog the Solar System, and to explore the transient optical sky. The catalogs generated by LSST during its ten years of operation will enable a multitude of science investigations beyond these primary science drivers, many of which are explored in the LSST Science Book (LSST et al. (2009)).

The inventory of the Solar System is one of the primary science drivers for LSST. Fulfilling this science goal will involve discovering millions of minor planets, increasing the number of known objects in every small body population by a factor of 10 to 100 above current levels. Many of these objects will receive large numbers $(>100)$ of observations, over a long time span (several years) and with extremely accurate astrometry (10mas 
Table 1. Summary of small body populations observed with LSST

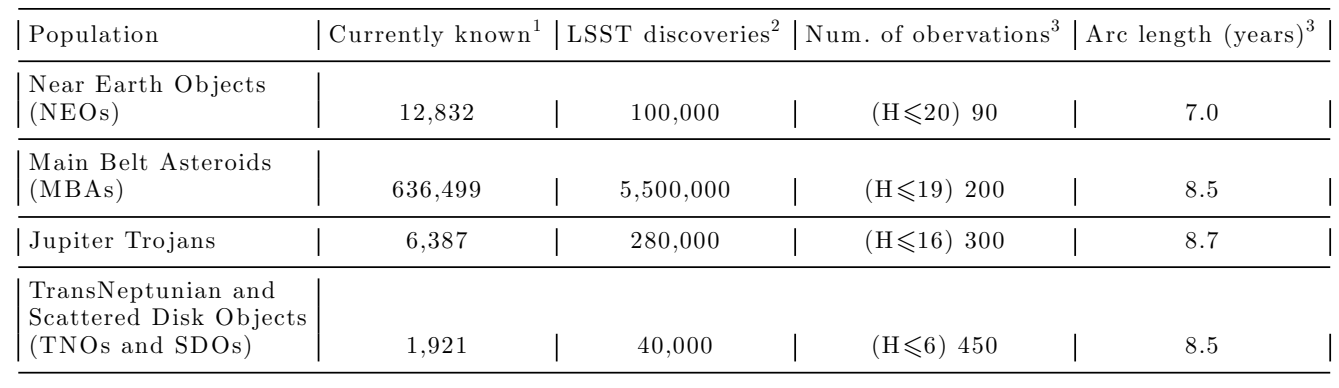

Notes:

${ }^{1}$ As reported by the MPC (May 2015). ${ }^{2}$ Expected at the end of LSST's ten years of operations. ${ }^{3}$ Median number of observations and observational arc length for the brightest objects near $100 \%$ completeness (as indicated).

errors), resulting in highly accurate orbits suitable for a wide range of theoretical studies or for targeted follow up observations for specific purposes (such as spectroscopy or occultation studies.

These large number of observations will also provide the basis for sparse lightcurve inversion, which requires at least 100 observations over a range of phase angles. It will be possible to determine the spin states and shapes for thousands of Main belt asteroids. Frequent observations, spread among a wide range of times and at variety of different points along each object's orbit, are also ideal for detecting activity, either collisionallyinduced activity or surface activity induced by volatile outgassing.

Each object will obtain observations in different filters, primarily $g, r, i$ and $z$ but also $u$ and $y$, with photometric calibration of each measurement accurate to 10mmags (Ivezić et al. (2008)). This will enable study of the composition of these objects. Adding color information also provides statistical constraints on the albedos of the objects, allowing a tighter estimate of the diameters and thus size distribution of the population. With combined color and orbital information, identification of collisional families becomes more robust. (See the Solar System chapter from LSST et al. (2009) for further discussion of these topics). Table 1 provides a summary of the expected number of objects in each population, as well as their typical arc length and number of observations.

Construction for LSST is ongoing, with first light scheduled for 2020, a scientific commissioning program following in the next year, and the start of survey operations in 2022. Details of LSST operations are currently being examined. In particular, the survey strategy continue to be analyzed up to and during operations in order to maximize the science return across the wide variety of goals for LSST. In this proceedings, we will describe the planned LSST configuration, and expected LSST performance in discovering and characterizing Near Earth Objects (NEOs) and Main Belt asteroids (MBAs), then present software tools that can aid the planetary astronomy community in extending this analysis.

\section{The LSST telescope}

The primary science goals for LSST drive the design of the telescope and camera. The choice of telescope mirror size, field of view, filters and typical exposure times combine to achieve the desired single image depth, coadded image depth, number of repeat visits, visit distribution among filters, and survey footprint.

The resulting final design is an optical telescope with ugrizy filters and a primary mirror of $8.4 \mathrm{~m}$ in diameter (the effective diameter is $6.5 \mathrm{~m}$ after accounting for obscuration 
Table 2. ugrizy $5 \sigma$ point source limiting magnitudes ${ }^{1}$

\begin{tabular}{|l|l|l|l|l|l|l|}
\hline & $u$ & $g$ & $r$ & $i$ & $z$ & $y$ \\
\hline Median atmospheric $\mathrm{IQ}^{2}$ & $\mid 0.66$ & $0.61 \mid$ & 0.56 & 0.53 & $0.50 \mid$ & 0.48 \\
\hline Dark sky brightness $\left(\mathrm{mag} / \mathrm{sq}^{\prime \prime}\right)^{3}$ & $\mid 23.0$ & 22.2 & 21.2 & 20.5 & 19.6 & $18.6 \mid$ \\
\hline $5 \sigma$ limiting magnitude & $\mid 23.6$ & 24.9 & 24.4 & 24.0 & 23.4 & $22.5 \mid$ \\
\hline
\end{tabular}

Notes:

${ }^{1}$ Please see the LSST Overview Paper (Ivezić et al. (2008)) for updated values. ${ }^{2}$ Based on Cerro Pachon site monitoring. ${ }^{3}$ Based on dark sky spectra convolved with the LSST bandpasses, validated with site monitoring data from Cerro Pachon.

and vignetting). The telescope has a fast $\mathrm{f} / 1.2$ focal ratio; together with the 3.2 Gigapixel camera, this provides a 9.6 square degree field of view with $0.2 \%$ / pixel platescale. Short exposures and a rapid survey strategy covering the entire visible sky every three to four nights in multiple filters complete the basic strategy to meet these science goals.

The details of the observing strategy will be discussed further in Section 4, but at the base of the cadence is the pair of back-to-back 15 second exposures that make up a 30 second 'visit'. For most purposes, this 30 second visit can be considered the equivalent exposure time for LSST; the back-to-back 'snaps' will be processed separately to help reject cosmic rays (and could be used to help determine velocity direction for trailing moving objects), but the images will be combined for most purposes and individual image depths correspond to the 30 second visit $5 \sigma$ point-source limiting magnitude. This drives further design choices for the telescope; in order to maintain a high duty cycle, the camera readout time is only 2 seconds per exposure and the slew-settle time between nearby fields is only 5 seconds per visit.

The fill factor of the camera is $90 \%$, counting active silicon within a $3.5^{\circ}$ diameter circle inscribed in the field of view; the fill factor counting only chip gaps but over the entire (non-circular) focal plane is slightly higher, but similar. See Figure 1 for an illustration of the focal plane.

On-site monitoring has provided information on the expected free atmosphere FWHM and sky brightness (see Table 2). The telescope hardware is expected to contribute an additional 0.4" to the delivered seeing. The expected dark sky skybrightness is generated using detailed sky spectra obtained elsewhere (Patat (2008)), modified to match broadband sky brightness measurements reported from Cerro Pachon and other nearby sites.

Expected throughput curves for each component of the hardware system are maintained by system engineering (see the github repo $\nmid$ for latest values). These are based on data from prototype sensors and the expected performance of the mirrors and filters and lenses, including broadband coatings and loss estimates due to condensation and contamination. The throughput curves for each filter are illustrated in Figure 2.

Combining all of the information above, we can calculate the expected five-sigma pointsource limiting magnitudes for LSST, under fiducial seeing and dark sky conditions - see Table 2.

As LSST continues to move toward operations, the expected values for each of these components will be replaced by 'as delivered' versions. Up-to-date values will be maintained in the github repositories and reported in the LSST Overview Paper (Ivezić et al. (2008)). 


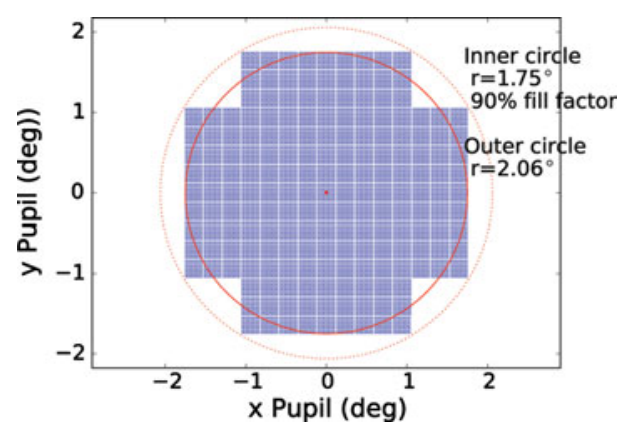

Figure 1. Layout of the LSST focal plane. The solid circle indicates the inscribed circular field of view $\left(3.5^{\circ}\right.$ diameter $)$. The plotted points indicate active silicon.

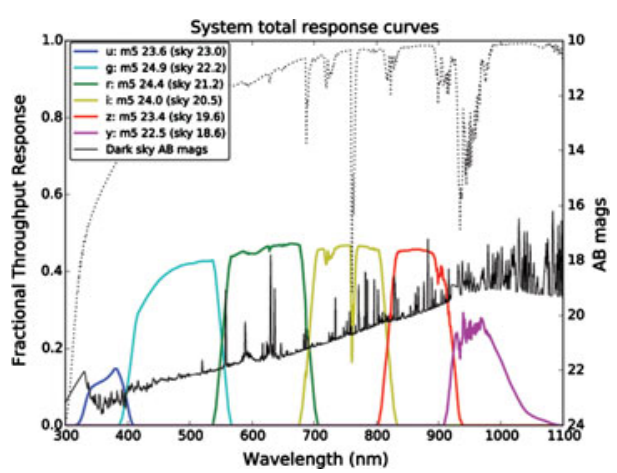

Figure 2. Expected LSST throughput response in ugrizy, including an atmospheric throughput curve (the dotted line). The expected dark sky brightness in $\mathrm{AB}$ magnitudes is also shown (the thin black line).

\section{LSST data management}

LSST will acquire millions of images - on the order of 2.5 million visits, each consisting of a pair of exposures. The LSST Data Management (DM) software pipeline has the task of turning these images into catalogs enabling the primary science goals. In general these catalogs can be thought of as falling into three categories: Level 1, Level 2 and Level 3.

Level 1 data products are created during nightly processing. The images in each visit are combined to reject cosmic rays, then subtracted from a template image created from previously acquired imaging (typically 6 months to a year earlier). The detections measured in each difference image correspond to transients, variables, moving objects, and artifacts. These outputs will be run through machine learning algorithms to help reject artifacts. The resulting detections, along with relevant information from existing catalogs such as identification of known variable stars or the location of nearby background galaxies, will be released within 60 seconds of the end of each visit as the LSST Alert stream.

In addition, these difference image catalogs (after removing known variable stars) will be used to feed the LSST moving object processing system (MOPS). MOPS will link detections from different visits within a night into tracklets, combine these with tracklets from other nights into tracks, and finally fit the tracks with orbits; it will also extend known orbits with new detections of these objects. These moving object catalogs will updated and released on a daily basis.

The Alert stream and the moving object catalogs (the linked orbits and their individual detections) make up the Level 1 data products. It is worth noting that moving objects which are measurably trailed in any individual visit will be clearly identifiable in the Alert stream as such; very fast-moving objects thus have an additional discovery avenue via Alerts.

Level 2 data products are created during yearly data processing and include a more precise level of calibration in photometry and astrometry. During the yearly data processing, all existing images will be reprocessed using the most recent software release (including reprocessing these images through MOPS, likely using slightly improved templates for image differencing). These data release catalogs will reach $10 \mathrm{mmag}$ absolute photometric accuracy and 10mas absolute astrometric accuracy. The increased accuracy 
is possible due to various algorithms that compute global solutions; these are not run during nightly data processing.

Level 3 data products indicate data products resulting from independently written (non-project) software, created using LSST data access center compute resources. These data products will typically be generated using extensions to the LSST DM software, and may or may be publicly available depending on the user. Publicly available Level 3 data products which prove particularly useful could become fully federated with LSST databases.

The LSST DM pipeline will be entirely open source and publicly available. The various repositories that make up the DM software stack can currently be found on github†; more information about the stack and instructions for installing the LSST software stack can be found at http://dm.1sst.org. Details of the data products (images and catalogs) are defined in the LSST Data Products Definitions Document (DPDD) $\ddagger$. All LSST data products will be immediately publicly available to institutions with data rights.

\section{LSST survey strategy}

The basic parameters of LSST - telescope size, field of view - have been fixed. In addition, given the survey length, visit exposure time, and constraints on the survey footprint, an approximate outer envelope of the survey characteristics can be estimated: the survey has about 2.5 million visits to distribute over about 25,000 square degrees for all survey fields, with about 825 visits per field in the main survey footprint $(\approx 18,000$ sq deg) to distribute among ugrizy filters. Most fields in the main survey footprint can be observed twice per night (with an interval of about 30 minutes) every three to four days, on an ongoing basis over their observing season, repeating for ten years. This is the strawman LSST observing strategy at present.

However, the details of the observing cadence have not yet been fixed. For example, instead of distributing visits fairly evenly in time for all fields over all ten years, a variant may be to concentrate a subset of those visits for some fields into a shorter period of time (a 'rolling cadence'). One option that may be interesting for studying solar system objects could include taking more frequent observations for fields near opposition and then reducing the number of observations for fields away from opposition. The process of optimizing the survey strategy in terms of cadence is just starting to get underway.

LSST has several tools to help this process of survey strategy optimization. The first is the LSST Operations Simulator (OpSim) (Delgado et al. (2014)), which combines a realistic weather history and a high-fidelity telescope model with a scheduler driven by a set of proposals that attempt to parametrize a basic observing strategy (e.g., a proposal for the main survey footprint that specifies the main survey footprint, skybrightness and seeing limits, number of visits desired in each filter, and the time window between pairs of visits in each night). The output of OpSim is a simulated pointing history, complete with observing conditions and individual visit limiting magnitudes, that demonstrate how LSST might survey the sky. A simple visualization of an OpSim run is shown in Figure 3; this also shows the footprint for the survey in various proposals and filters.

The second tool is a user-extensible python package called the LSST Metrics Analysis Framework (MAF) (Jones et al. (2014)). MAF was created to help analyze OpSim outputs. Using MAF, it is simple to write short pieces of python code ('metrics') that can be plugged into the framework to evaluate some aspect of OpSim. By collecting these

$\dagger$ http://github.com/lsst

$\ddagger$ http://www.lsst.org/content/data-products-definition-document 

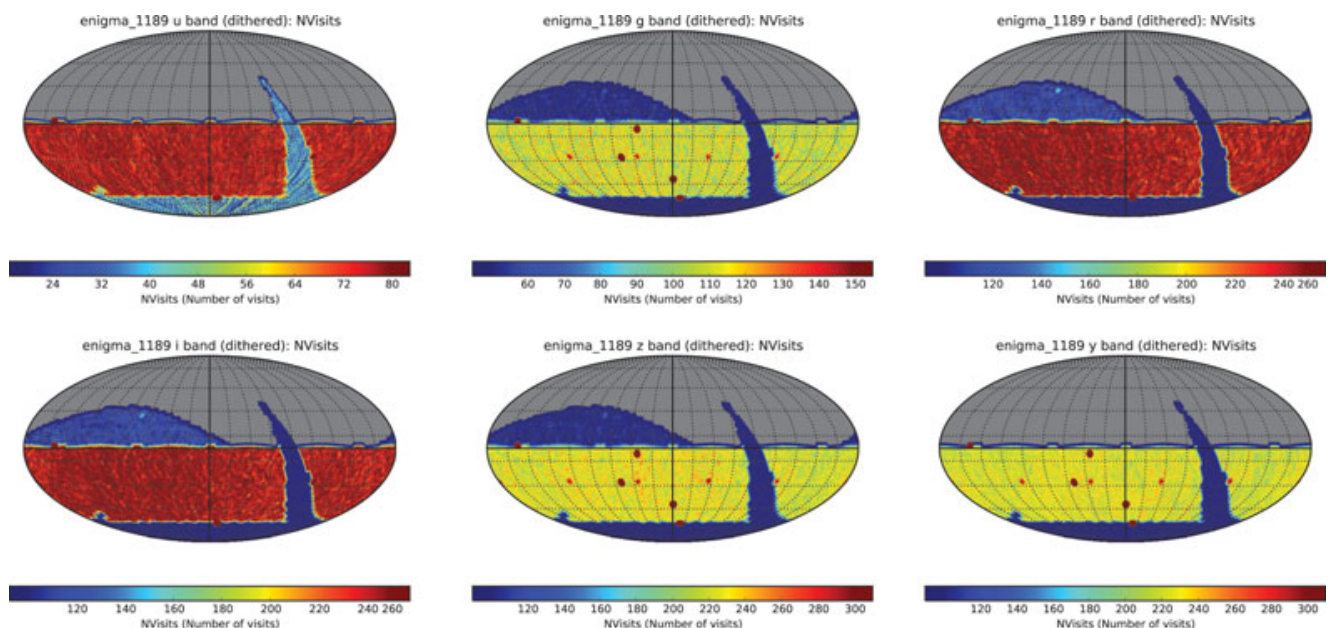

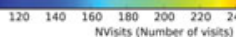

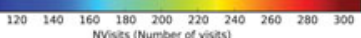

Figure 3. The distribution of visits across the LSST survey footprint, in a sample OpSim simulated survey. The main survey covers the area from $-65^{\circ}<$ Dec $<5^{\circ}$, excluding a small area around the galactic plane. The area from Dec $=5^{\circ}$ up to $10^{\circ}$ north of the ecliptic is covered in an additional observing program; other programs cover the South Celestial Pole and the Galactic Plane.
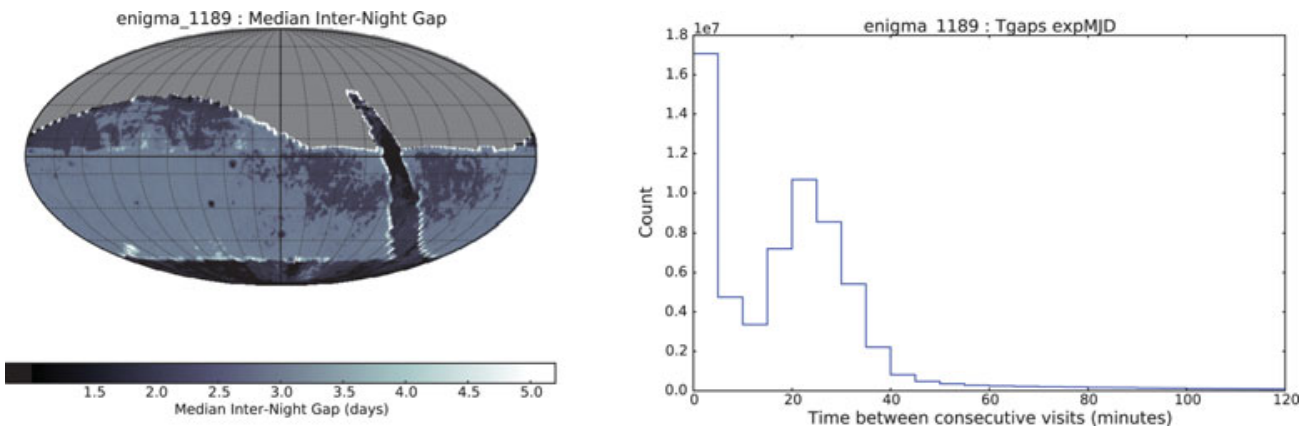

Figure 4. Left: the median number of nights between consecutive visits to a field, for an OpSim simulated survey. Right: A histogram of revisit times, within each night.

metrics from a wide representation of the astronomical community, we can evaluate OpSim surveys created with a variety of scheduler configurations and maximize science return from LSST across a wide range of science goals. An example of using MAF to evaluate the median time between revisits at each point on the sky is given in Figure 4.

OpSim and MAF are open-source software packages, provided as part of the LSST Systems Engineering Simulations effort. Instructions for installing them are available at online†.

\section{Evaluating the LSST survey strategy for Solar System science}

The LSST Metrics Analysis Framework (MAF) can also be used to evaluate the performance of OpSim simulated surveys with respect to Solar System science goals. MAF will allow a user to specify a population of moving objects (by providing their orbital parameters), specify a particular OpSim survey, and then generate their simulated observations.

$\dagger$ https://confluence.lsstcorp.org/display/SIM/Catalogs+and+MAF 
MAF uses the open-source package OpenOrb (Granvik et al. (2009)) in generating the ephemeris information for these simulated observations.

In many cases, the input population of moving objects can be small (on the order of a few thousand); MAF is able to clone the resulting detections over a range of $H$ magnitudes, so that metrics can be evaluated over a wide range of $H$ while only using a relatively small set of orbits. MAF tests using 10,000 MBAs produced identical metric results as tests using 2,000 MBAs; statistically, this method of cloning the input population is adequate for most purposes.

As MAF generates the detection lists for each object, the reference $H$ value in the orbit file is used to generate an apparent $V$ band magnitude, then the (optionally user-assigned) spectrum is used to generate a magnitude in the LSST bandpass. When the object is cloned over the user-specified range of $H$, these apparent magnitudes are adjusted accordingly. When evaluating a specific metric (e.g. the number of observations obtained for each orbit in the sample for a range of $H$ magnitudes), the desired SNR cutoff can be specified and calculated, including trailing losses and the $5 \sigma$ limiting magnitude for each visit.

Trailing loss estimates are provided by MAF. Trailing losses occur whenever the motion of a moving object spreads their light over a wider area than a simple stellar PSF. There are two aspects of trailing loss to consider: simple SNR losses and detection losses. The first is simply the degradation in SNR that occurs (relative to a stationary PSF) because the trailed object includes a larger number of background pixels in its footprint. This will affect photometry and astrometry, but typically doesn't directly affect whether an object is detected or not. The second effect (detection loss) is not related to measurement errors but does typically affect whether an object passes a detection threshhold. Detection losses occur because source detection software is optimized for detecting point sources; a stellar PSF-like filter is used when identifying sources that pass above the defined threshhold, but this filter is non-optimal for trailed objects. This can be mitigated with improved software (e.g. detecting to a lower SNR threshhold and attempting to detect sources using a variety of trailed PSF filters). Both trailing losses can be fit as:

$$
\begin{aligned}
\Delta m & =-1.25 \log _{10}\left(1+\frac{a x^{2}}{1+b x}\right) \\
x & =\frac{v T_{\text {exp }}}{24 \theta}
\end{aligned}
$$

where $v$ is the velocity (in degrees/day), $T_{\text {exp }}$ is the exposure time (in seconds), and $\theta$ is the FWHM (in arcseconds). For SNR trailing losses, we find $a=0.67$ and $b=1.16$; for detection losses, we find $a=0.42$ and $b=0$. An illustration of the magnitude of these trailing loss effects for 0.7" seeing is given in Figure 5. When considering whether a source would be detected at a given SNR using typical source detection software, the detection loss should be used.

MAF can also include the details of the camera focal plane layout, as illustrated in Figure 1; detections which would fall into chip gaps are then removed.

To demonstrate the potential of LSST as a tool for studying the Solar System, we calculate a variety of metrics for a set of small body population samples ranging from Potentially Hazardous Asteroids (PHAs) to TransNeptunianObjects (TNOs). The 2000 sample orbits used for each population except the PHAs come from the Pan-STARRS Synthetic Solar System Model Grav et al. (2011); the PHA orbits are taken from the Minor Planet Center $\dagger$ database, trimmed to the subset of $\approx 1500$ objects larger than

$\dagger$ http://www.minorplanetcenter.net/ 


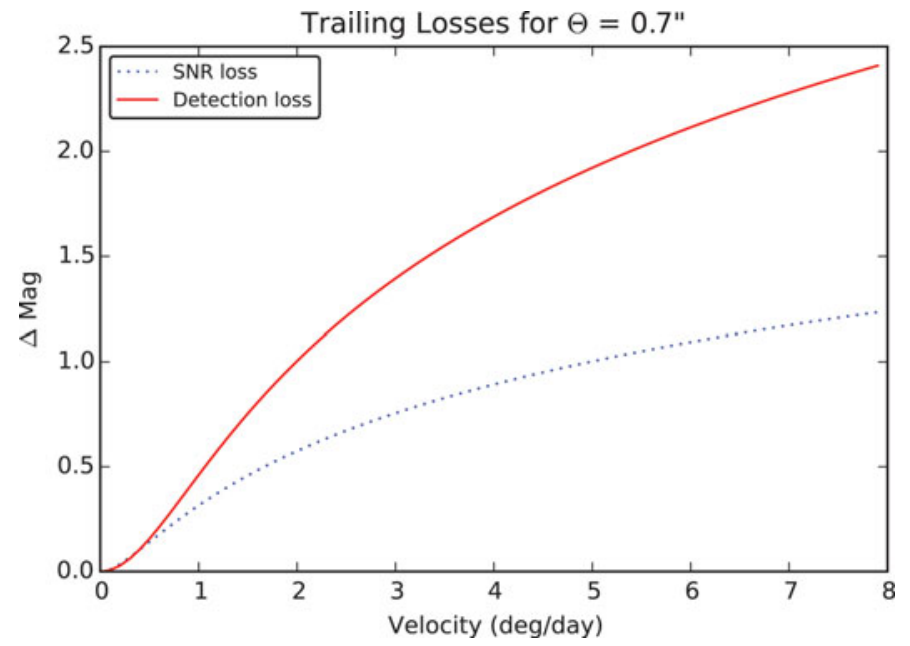

Figure 5. Trailing losses for 30 second LSST visits, assuming seeing of 0.7 ". The dotted line shows SNR trailing losses, the solid line indicates detection trailing losses. With software improvements detection losses can be mitigated.

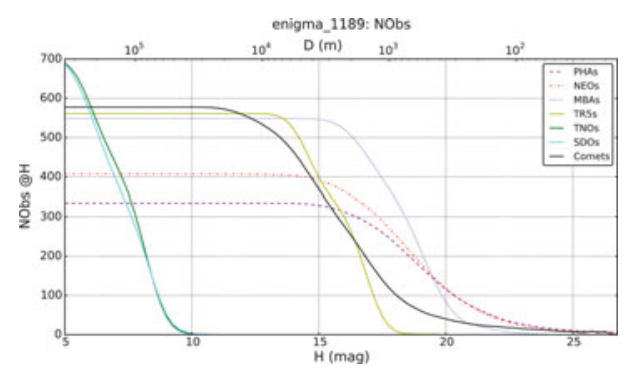

Figure 6. The mean number of observations (per object) for each of our sample small body populations, as a function of $H$ magnitude.

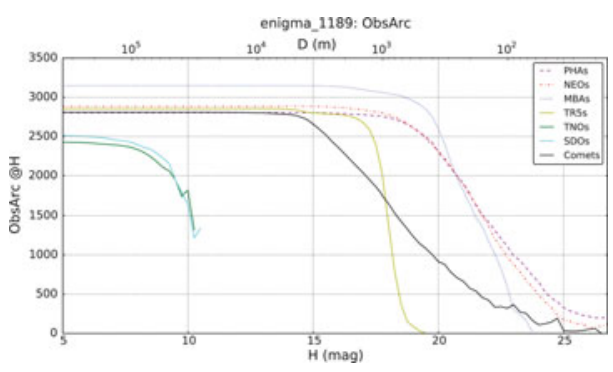

Figure 7. The mean observational arc (in days) for each of our sample small body populations, as a function of $H$ magnitude.

$1 \mathrm{~km}$ in diameter. In all metrics shown here, we then clone these orbits over a range of $H$ magnitudes, assumed the (larger) detection trailing loss, included the camera focal plane footprint, and only used resulting detections above a $\mathrm{SNR}=5$ cutoff.

First we simply count the total number of observations for each orbit as a function of $H$; the mean value for all orbits in each population is shown in Figure 6. Similarly, we can look at the time of the first and last observation to get the overall observational arc length; the mean values of the observational arc are shown in Figure 7.

We also calculate the number of 'discovery opportunities' available for each object and use this to calculate the overall completeness across the population (counting an object as 'discovered' if it had at least one discovery opportunity). The definition of a discovery opportunity can be varied by the user, but here we look at a variety of cases. First, the current basic MOPS requirement: detections on three different nights within a window of 15 nights, with detections in at least two visits per night separated by less than 90 minutes. Second, an extended MOPS requirement intended to be more rigorous while still nominally matching the typical observing pattern in our OpSim survey: detections on four different nights within a window of 20 nights, with at least two visits per night. Third, a relaxed discovery criteria intended to demonstrate the effect of improving MOPS 

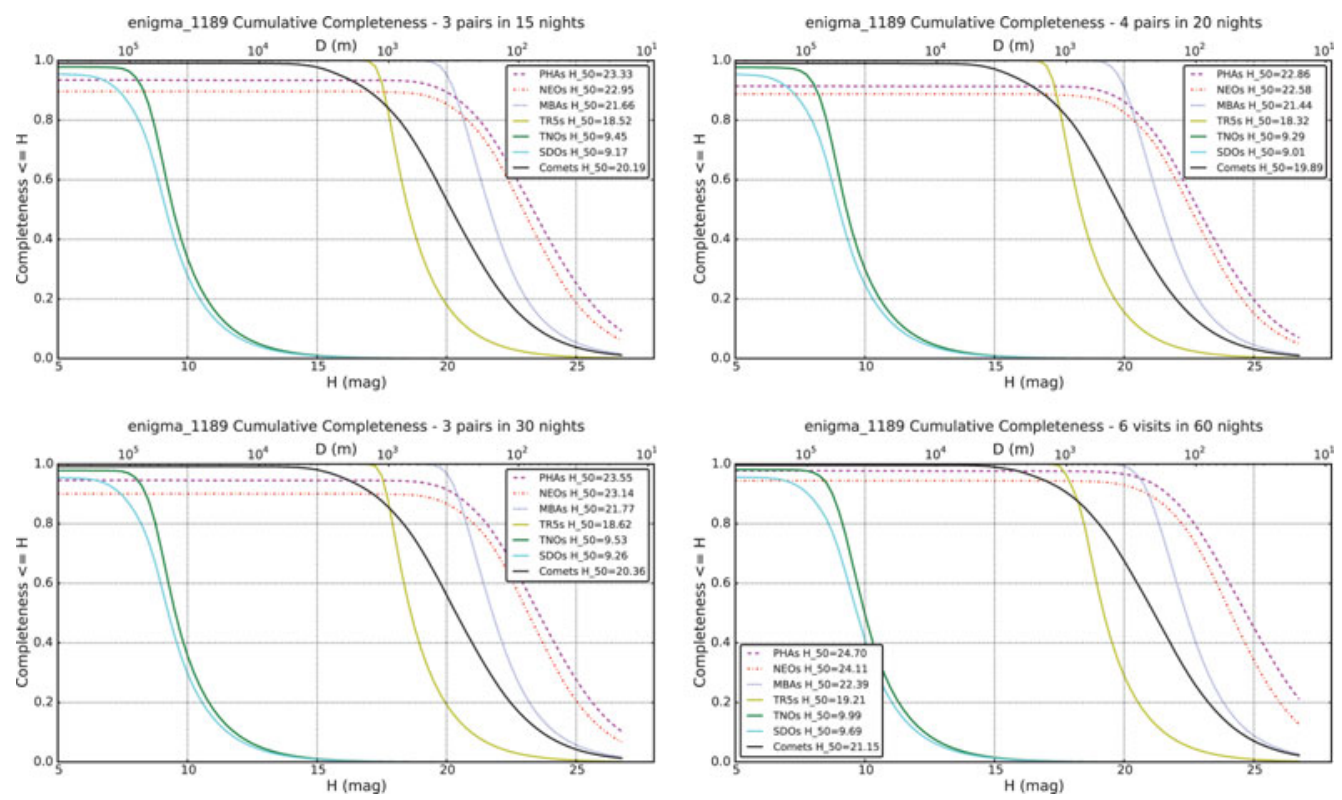

Figure 8. Cumulative completeness calculated using various discovery criteria as outlined in the text, for each of our sample small body populations. $H_{50}$ in the plot legends indicates the $H$ value where the cumulative completeness falls to $50 \%$ of the overall population.

software: 3 nights within 30 days, again with at least 2 visits per night. Finally, a 'magic' discovery criteria intended to get an idea of the upper limit for detection if linking software is not a constraint: 6 observations in 60 nights. The cumulative completeness (completeness for $H \leqslant X$ ) is calculated by multiplying the differential completeness values by a power law $(\alpha=0.3)$. The results are shown in Figure 8 , including the value $H_{50}$, corresponding to the $H$ value where the cumulative completeness drops to $50 \%$ of the overall population. It can be see that these varying discovery scenarios have the largest effects on the PHA and NEO populations. With more rigorous requirements, the $H_{50}$ values are increased by a few to several tenths of a magnitude; with relaxed requirements, these values are pushed faintward by a few tenths. The peak completeness levels change by a few percent for PHAs and NEOs only. This suggests that even with the basic cadence LSST is doing fairly well at discovering moving objects; with improvements in the cadence (probably some version of a rolling cadence to concentrate more visits into a given chunk of time) it could do better; and money spent on improving linking software, even by a relatively modest amount, directly leads to improvements in completeness.

To demonstrate a more specialized MAF metric, aimed at evaluating the capability of LSST to help determine the source of activity in active asteroids, we also present the result of a 'activity detection' metric. Here we take the detections of each object after applying all the focal plane and SNR cuts and bin the times of these detections according to either time since the start of the survey (interesting if activity is due to collisions and thus random) or by time relative to the period of the object (the interesting timescale if activity is periodic and associated with the object's orbit). We then have calculated the probability of observing the object on a given timescale, and if we assume LSST DM will provide enough information to identify activity if it is present, this is equivalent to the probability of detecting activity on that given timescale. Repeating this exercise over a variety of timescales, we come up with the probabilities of detecting activity shown in Figures 9 and 10. 


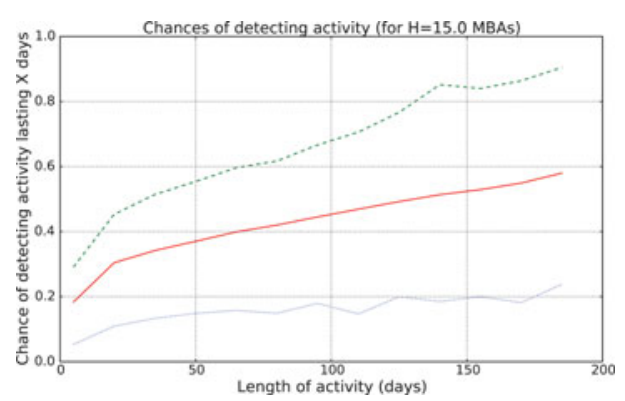

Figure 9. The likelihood of detecting activity which lasts at least a given amount of time (in days); mean (solid) probabilities across each of the sample populations, as well as maximum (dashed) and minimum (dotted) probabilities for individual objects within the sample.

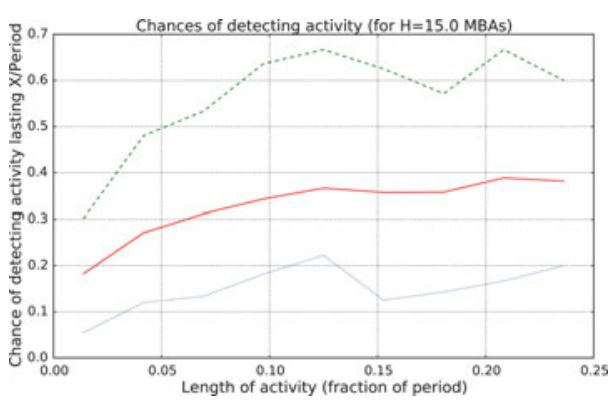

Figure 10. The likelihood of detecting activity which lasts at least a given fraction of the orbital period of the object; mean (solid) probabilities across each of the sample populations, as well as maximum (dashed) and minimum (dotted) probabilities for individual objects within the sample.

\section{Discovering moving objects with MOPS}

The discovery requirement with the LSST Moving Object Pipeline System (MOPS) is: detections on least three separate nights within a 15 night window, with at least two detections (visits) in each night, separated by 15 to 90 minutes. The detections within each night are joined into tracklets, and then tracklet detections from multiple nights are linked into tracks, which can then be fed to orbit determination algorithms to filter true from false linkages.

Tests of prototype LSST MOPS with these requirements and an additional constraint that the velocity on each night must be below 0.5 degrees/day, running on modest hardware (16 cores, $<20 \mathrm{~GB}$ of RAM) showed that in the absence of noise, moving object detections at the full depth and cadence of LSST could be easily linked together into tracks. These tests were repeated with increasing levels of random noise in the input detection lists. At a ratio of 4:1 noise:real detections, MOPS was still successful in creating tracks from the input catalogs; although the software compute requirements (runtime and memory usage) increased, there was no significant loss in terms of found objects $\dagger$.

The noise, or false positive rate of difference image detections, is a crucial consideration for MOPS. Reducing the false positive rate places high quality demands on the camera and optical system to reduce defects and ghosting and on the difference image software to reduce artifacts. Prototype LSST sensors have been delivered and are testing within specification and are cosmetically clean. Amp-amp crosstalk is well within specifications, and CCD-CCD crosstalk is too small to be measurable. Tests have not shown any measurable charge persistence under expected operating conditions. The optical system has been extensively modeled and has extremely small optical ghosting over the full focal plane. LSST is investing a significant amount of effort into its difference image software, both for moving object detection and for the purposes of the Alert pipeline. Existing surveys such as the Palomar Transient Factory and the Dark Energy Survey are already using advanced difference image pipelines on cosmetically clean and well characterized systems to achieve false positive rates of 13:1 (noise:real); with the addition of machine-learning algorithms to filter artifacts, these pipelines can achieve rates of 1:3 noise:real detection rates (Goldstein et al. (2015)). Existing surveys are already achieving false-positive rates within the acceptable range of our prototype MOPS.

$\dagger$ https://github.com/lsst/mops_daymops/blob/master/doc/report2011/LDM-156.pdf 
Work is ongoing to understand the limitations and capabilities of MOPS, and the prototype MOPS software will also be improved prior to survey operations.

\section{Conclusion}

The catalogs of minor planets that will come from LSST over its lifetime have enormous potential for planetary science. Small body populations throughout the Solar System will see an increase of 10-100 times more objects than currently known, including Earth minimoon, irregular satellite, and cometary populations. Many of these new objects will have large numbers of observations over the course of several years, in multiple filters, allowing for scientific characterization of the physical properties of these populations.

LSST provides simulation tools (OpSim and MAF) to assess the impact of the survey strategy on specific science goals. We encourage feedback from the community, especially in terms of metrics, to help maximize the scientific return of LSST. Further development and evaluation of LSST DM pipelines is ongoing, particularly in the areas of difference imaging and MOPS. First light for LSST is in 2020, with survey operations starting 2022.

\section{References}

Delgado, F., Saha, A., Chandrasekharan, S., Cook, K., Petry, C., \& Ridgway, S. 2014, in Modeling, Systems Engineering, and Project Management for Astronomy VI, Proc. SPIE 9150, 915015.

Denneau, L., Jedicke, R., Grav, T., Granvik, M., Kubica, J., Milani, A., Vereš, P., Wainscoat, R., Chang, D., Pierfederici, F., Kaiser, N., Chambers, K. C., Heasley, J. N., Magnier, E. A., Price, P. A., Myers, J., Kleyna, J., Hsieh, H., Farnocchia, D., Waters, C., and others 2013, $P A S P, 125,357$.

Granvik, M., Virtanen, J., Oszkiewicz, D., \& Muinonen, K. 2009, Meteoritics and Planetary Science, 44, 1853.

Grav, T., Jedicke, R., Denneau, L., Chesley, S., Holman, M. J., \& Spahr, T. B. 2011, PASP, 123,423 .

Goldstein, D. A., D'Andrea, C. B., Fischer, J. A., Foley, R. J., Gupta, R. R., Kessler, R., Kim, A. G., Nichol, R. C., Nugent, P. E., Papadopoulos, A., Sako, M., Smith, M., Sullivan, M., Thomas, R. C. and others 2015, AJ, 150, 82.

Ivezić, Ž., Tyson, J. A., Acosta, E., Allsman, R., Anderson, S. F., Andrew, J., Angel, J. R. P., Axelrod, T. S., Barr, J. D., Becker, A. C. and others 2008, arXiv:0805.2366v4.

Jones, R. L., Yoachim, P., Chandrasekharan, S., Connolly, A. J., Cook, K. H., Ivezic, Ž., Krughoff, K. S., Petry, C., \& Ridgway, S. T. 2014, in Modeling, Systems Engineering, and Project Management for Astronomy VI, Proc. SPIE 9149, 91490B.

LSST Science Collaborations and LSST Project 2009, LSST Science Book, Version 2.0, arXiv:0912.0201.

Patat, F. 2008, A\&A, 481, 575. 